\title{
Modelos in situ para Evaluar Potencial Erosivo de Bebidas
}

\author{
in situ Models to Evaluate Erosive Potential of Beverages
}

\author{
Shareen Melannie Acuña Flores; Lidia Yileng Tay Chu-Jon \& Leyla Delgado-Cotrina
}

\begin{abstract}
ACUÑA, F. S. M.; TAY-CHU-JON, L. Y. \& DELGADO-COTRINA, L. Modelos in situ para evaluar potencial erosivo de bebidas. Int. J. Odontostomat., 15(3):788-794, 2021.

RESUMEN: Existen varias metodologias in vitro, in situ e in vivo para evaluar el potencial erosivo de diferentes bebidas como jugos de frutas naturales, jugos procesados, bebidas alcohólicas, bebidas funcionales o productos y técnicas para el control de esta patología. Los modelos in situ permiten que los tejidos evaluados se desarrollen en un entorno natural, en presencia de saliva y película adquirida; además los especímenes pueden ser retirados para analizar sus propiedades mecánicas, físicas y biológicas de manera cuantitativa y cualitativa. El objetivo de esta revisión de literatura fue presentar los diferentes modelos in situ para evaluar el potencial erosivo de las bebidas sobre tejidos dentarios. Se desarrollarán los criterios para la selección de participantes, sustrato, dispositivos intraorales, agentes erosivos, ciclos erosivos y ensayos o test empleados.
\end{abstract}

PALABRAS CLAVE : erosión de los dientes; bebidas; in situ.

\section{INTRODUCCIÓN}

La pérdida de estructura dental por erosión dental o desgaste dental erosivo, que afecta en general a niños, adolescentes y adultos, se ha incrementado debido a cambios de estilos de vida relacionados a hábitos alimenticios, medicación u otros que condicionan a la acción química de ácidos (Schlueter \& Luka, 2018; Vieira Pedrosa \& de Menezes, 2020). El potencial erosivo de los alimentos y bebidas que tienen contacto con los dientes depende de la acidez titulable, el tipo de ácido, $\mathrm{pH}$, concentración de calcio, fosfato y fluoruro, sin embargo, para que ocurra erosión dental debemos considerar factores de comportamiento como la forma de ingerir, exposición nocturna, prácticas de higiene oral y factores biológicos como la capacidad buffer, rango de flujo salival y formación de película adquirida (Buzalaf et al., 2018; Acuña et al., 2020; Chan et al., 2020; Warreth et at., 2020).

Se han validado una serie de metodologías para la evaluación de la pérdida de tejido producido por erosión. Idealmente los estudios deberían ser in vivo, cuya ventaja es evaluar la pérdida de tejido den- tal intraoralmente en condiciones reales, pero no siempre es posible ejecutar estos estudios debido a la incertidumbre sobre el patrón de la progresión en estudios a largo plazo, años de seguimiento y la falta de control sobre la exposición al desgaste además de la necesidad de minimizar el contacto de las sustancias erosivas con la dentición de los voluntarios a prueba; por lo tanto se han desarrollado modelos in vitro e in situ (West et al., 2011; Shellis et al., 2011; Huysmans et al., 2011; Young \& Tenuta, 2011; Wiegand \& Attin, 2011).

Los estudios in vitro permiten exponer muestras, de esmalte o de dentina de dientes extraídos, a un producto y se cuantifica sus efectos en un laboratorio (West et al., 2011; Shellis et al.).El modelo in situ es un protocolo intermedio entre in vivo e in vitro que permite evaluar el efecto de un agente erosivo sobre muestras que pueden ser retiradas y analizadas en laboratorio y que han estado en condiciones intraorales como el desarrollo de la película adquirida, capacidad amortiguadora de la saliva, dilución de 
agentes erosivos y efecto remineralizante generando resultados más relevantes desde el punto de vista clínico (West et al., 2011; Shellis et al.; Huysmans et al.; Young \& Tenuta; Wiegand \& Attin, 2011; Attin \& Wegehaupt, 2014).

La presente revisión de literatura tiene como objetivo mostrar los diferentes modelos in situ para evaluar el potencial erosivo de las bebidas sobre tejidos dentarios.

Fuentes de información. La información de la revisión de la literatura se obtuvo de las bases de datos: Pubmed, SciELO y Scopus. Las palabras claves empleadas fueron: dental erosion, tooth erosion, in situ, beverages y sus combinaciones. Se incluyeron estudios experimentales de erosión in situ, artículos de revisión y revisiones sistemáticas. No se limitó la búsqueda por fecha de publicación.

\section{EVALUACIÓN IN SITU}

Criterios para la selección de participantes. La participación de voluntarios requiere la aprobación del protocolo por un comité de ética y la aceptación por escrito de un consentimiento informado antes de participar (West et al., 2011; Shellis et al.; Huysmans et al.).El número de participantes utilizados en los estudios in situ oscila entre 12 a 20 voluntarios (Hara et al., 2006; Turssi et al., 2014; Wiegand \& Attin, 2014; Almeida, 2016; Ablal et al., 2017; João-Souza et al., 2017; da Silva et al., 2017; de Souza et al., 2018; Santos et al., 2018; Kensche et al., 2019; Rios et al., 2019). El cálculo del tamaño de muestra se realiza a partir de un estudio piloto o de resultados de estudios anteriores, y debe ser suficiente para dar al estudio un poder estadístico adecuado considerando además el $20 \%$ de deserción de los voluntarios (Hara et al., 2014; Almeida; Mendonça et al., 2017; Santos et al.; Mullan et al., 2018; Mendonça et al., 2019). Dependiendo del estudio, se recomiendan participantes entre 18 a 30 años para evitar la influencia de otros factores como problemas oclusales mayores, ingesta de medicamentos u otros que puedan influir en los resultados, siempre y cuando la edad no sea una variable del estudio (West et al., 2011; Shellis et al.; Huysmans et al.).

Es importante estandarizar ciertas condiciones en los participantes como flujo salival estimulado (> $1 \mathrm{ml} / \mathrm{min}$ ) y no estimulado (>0,1 $\mathrm{ml} / \mathrm{min}$ ) además de una adecuada salud oral. Para la evaluación del flujo salival estimulado y no estimulado se recolecta saliva 2 horas después de la última comida. Para la saliva estimulada, los voluntarios mastican parafina durante un minuto, luego se recolecta la saliva por 5 minutos (Acuña et al.). Es importante descartar toda condición que pueda interferir en los resultados del estudio como presencia de lesiones de caries, erosión, gingivitis o periodontitis, enfermedades sistémicas como diabetes tipo 1, malnutrición, problemas gastroesofagicos, vómito, embarazo o lactancia, uso de aparatología ortodóncica, uso de medicamentos que afectan el flujo salival como antidepresivos, antidiuréticos y fluoruros en los últimos 2 meses (Hara et al., 2014; Almeida; Mendonça et al., 2017, 2019; Mullan et al.)

Se les indica a los voluntarios que no coman ni beban mientras usan el dispositivo en boca, ya que la alimentación variada entre ellos puede alterar los resultados; además, se les indica no pasar la lengua por las superficies de los especímenes porque incrementa la pérdida de tejido (Mullan et al.).

Sustrato. Para comprender mejor los procesos involucrados en la erosión dental y poder revertir su efecto, se utilizan dientes extraídos, generalmente especímenes de esmalte o dentina de premolares 0 terceros molares permanentes (Hooper et al., 2014; West et al., 2019) o esmalte de dientes deciduos humanos (Scatena et al., 2018; Magalhães et al., 2009).

Es importante considerar la edad del donante de los dientes; se ha demostrado que el esmalte dental natural maduro es menos susceptible a desafíos erosivos que el esmalte inmaduro ya que existen diferencias en el contenido mineral y las características de la superficie (West et al., 2011; Young \& Tenuta; Yassen et al., 2011).Es así que se prefieren utilizar dientes premolares que terceros molares para este tipo de estudios, además es necesario la estandarización cuidadosa de la preparación de las muestras y la asignación al azar de las mismas, entre los participantes. Existen diferencias fisiológicas del esmalte y la dentina entre sujetos, entre dientes en la misma boca y entre diferentes partes del mismo diente, es por eso que las muestras de los estudios in situ deben ser pulidas para compensar parcialmente estas diferencias (West et al., 2011; Shellis et al.; Wiegand \& Attin, 2011; Yassen et al.).

También es frecuente encontrar estudios que emplean dientes de origen bovino, por su facilidad de obtención (Yassen et al.). Se ha demostrado que los resultados en dentina humana y bovina son similares 
(Moda et al., 2019), sin embargo, en esmalte, se ha observado que la velocidad de la pérdida de tejido y tasa de desmineralización es mayor en esmalte bovino que humano (West et al., 2011; Amaechi et al., 1999), y en estudios in situ el esmalte bovino mostró una microdureza significativamente menor comparado con el esmalte humano (Rios et al., 2006, 2008); a pesar de este hallazgo, Turssi et al. (2010) no encontraron diferencias significativas entre los valores de pérdida de microdureza del esmalte humano y bovino. Field et al. (2017) compararon in vitro esmalte humano, bovino y ovino sometido a desafío erosivo y abrasivo; encontraron que el esmalte ovino mostró poca correlación en dureza y rugosidad superficial con el esmalte humano, mientras que el esmalte bovino mostró tendencias similares, sin embargo, al aumentar la concentración del ácido, la pérdida de minerales es significativamente mayor en el esmalte bovino.

Así, estudios en esmalte bovino nos pueden mostrar la misma tendencia de pérdida de minerales en el esmalte humano pero los valores serán diferentes, por lo que sí es posible obtener especímenes humanos debe preferirse o se debe hacer un analísis exhaustivo de los resultados obtenidos. Por otro lado, si queremos evaluar lo que ocurre en la dentina, la dentina bovina sí responde de manera similar a la dentina humana (Yassen et al.; Rios et al., 2006, 2008; Turssi et al., 2010; Field et al.).

Los sustratos deben ser sometidos a procedimientos de desinfección y esterilización para garantizar especímenes estériles y seguros para los pacientes. Entre estos procedimientos se han utilizado con mayor frecuencia: solución acuosa de timol al 0,1\% (Hara et al., 2006; Turssi et al., 2010, 2014; Wiegand \& Attin, 2014; Hara et al., 2014; Almeida; João-Souza et al.; Scatena et al.; de Souza et al.), clorhexidina al 0,5\% durante 30 minutos (West et al., 2004; Hooper et al., 2007, 2014; Field et al.; West et al., 2019), 2 \% de formaldehído durante 30 días a $\mathrm{pH} 7$ para la desin- fección de los dientes antes de su uso in situ (Rios et al., 2006, 2008; Magalhães et al.; Rios et al., 2009) y para la esterilización óxido de etileno durante una hora a $55^{\circ} \mathrm{C}$ (Hara et al., 2006; Hooper et al., 2007; Turssi et al., 2010; Hara et al., 2014; Turssi et al., 2014; Almeida; Ruben et al., 2017; de Souza et al.; Santos et al.; Mendonça et al., 2019; Jordão et al., 2019; Rios et al., 2019). La esterilizacion con óxido de etileno es un procedimiento eficaz para dientes extraidos que no altera la dureza superficial, la respuesta a la desmineralización del esmalte y es de fácil acceso ya que es ampliamente utilizado en la esterilización de instrumental médico (Hara et al., 2006; West et al., 2011; Shellis et al.; Wiegand \& Attin, 2011).

Dispositivos intraorales. Los dispositivos se utilizan para poder exponer los especímenes de estudio al medio oral del participante; este debe adaptarse cómodamente a la boca del paciente y permitir la fijación de los especímenes durante un periodo de tratamiento (West et al., 2011). Se colocan entre 1 a 8 especímenes en una placa, de tal modo que permita su retiro y reemplazo para poder evaluarlos. Estos pueden ser dispositivos fijos, removibles o simulando una prótesis total en el caso de pacientes edéntulos (West et al., 2011; Shellis et al.; Huysmans et al.; Young \& Tenuta Wiegand \& Attin, 2011; Ruben et al). Los datos obtenidos de las diferentes pruebas cuantitativas de los especímenes en un mismo dispositivo no difieren, es por eso que los últimos estudios disminuyen la cantidad de especímenes por dispositivo (Rios et al., 2019; Kenshche et al.; de Souza et al.; Santos et al.; Mendonça et al., 2019; West et al., 2019; Jordão et al.).

Dispositivos Fijos. En este caso los especímenes son fijados con composite ortodóntico a los dientes de los voluntarios por periodos cortos y se puede usar protectores orales de acetato transparente con ventanas que permite la protección de los dientes del participante durante la toma de la bebida de prueba (Amaechi \& Higham, 2001; West et al., 2011). (Fig. 1). Cuando
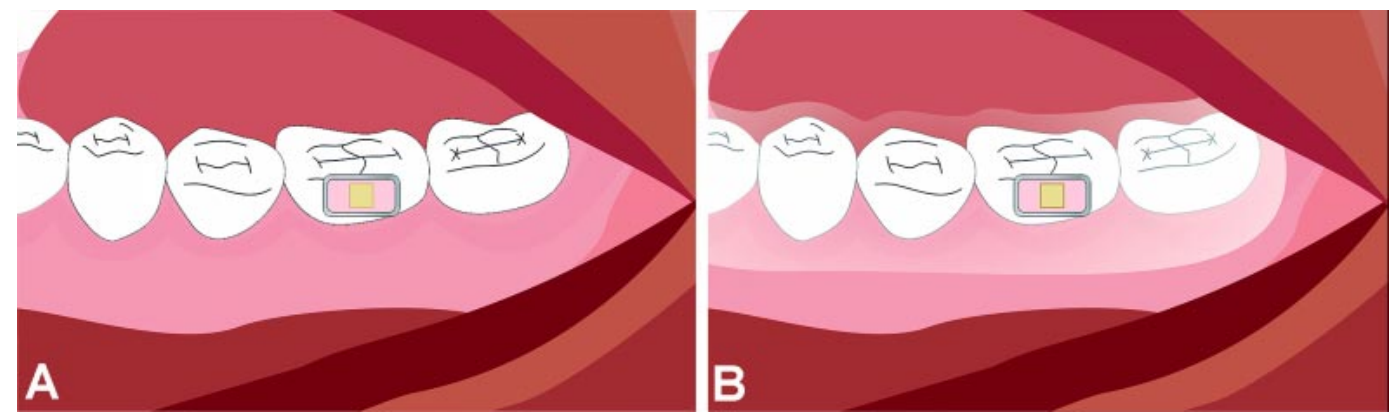

Fig. 1. Dispositivo fijo a. Especimen fijado a primer molar inferior b. Protector oral 
los especímenes son fijados sin protectores de acetato, el investigador se asegura de que los especímenes estén expuestos todo el tiempo al medio oral (West et al., 2011; Shellis et al.), sin embargo existe la posibilidad producir una pérdida de minerales en los dientes del voluntario, susceptibilidad a la abrasión debido a la exposición a fuerzas mecánicas de los tejidos blandos y lengua (Amaechi et al., 2003; Gregg et al., 2004; Mullan et al.). Además de ser un factor de retención de placa bacteriana (West et al., 2011; Shellis et al.; Huysmans et al.; Young \& Tenuta; Jordão et al.).

Dispositivos removibles. Generalmente, son de acrílico autopolimerizable, con retenedores adams en los primeros molares (West et al., 2011). Presentan cavidades donde los especímenes son fijados con cera dental, posteriormente se coloca un alambre de ortodoncia que pasa por encima de los especímenes pero sin tocarlos para proteger de la abrasión de la lengua y tejidos blandos (West et al., 2011; Ablal et al.). Su uso puede ser intermitente o continuo. El uso intermitente del dispositivo se da bajo supervisión, son retirados en horarios de alimentación y durante la noche (West et al., 2011; Shellis et al.; Huysmans et al.; Young \& Tenuta; Wiegand \& Attin, 2011), permite que la investigación sea más estandarizada bajo condiciones estrictas minimizando la influencia del comportamiento del participante, sin embargo al no utilizar el dispositivo en la noche se deben mantener las muestras en saliva artificial o en una solución de agua destilada (West et al., 2011). En el uso continuo se mantiene el dispositivo durante la noche, retirándose de la cavidad oral solo durante horarios de alimentación (West et al., 2011; Shellis et al.), permite simular situaciones en condiciones reales.

Los dispositivos pueden ser palatinos y mandibulares. En los palatinos, los especímenes se pueden ubicar centralmente en la línea media (West et al., 2011) o hacia los lados derecho e izquierdo o anterior y posterior (Jordão et al.) (Figs. 2a-e) Además se puede analizar varios grupos de estudio en un mismo dispositivo con el diseño de boca dividida, como por ejemplo colocar diferentes especímenes (esmalte/dentina) en la misma placa palatina o someter a diferentes ciclos erosivos o sustancias las muestras de cada hemiarcada (Acuña et al.; Young \& Tenuta; Magalhães et al.).

Los dispositivos mandibulares tienen una cavidad a cada lado para la fijación de los especímenes, pueden colocarse a nivel vestibular o lingual (Jordão et al.). (Figs. 2f-g).

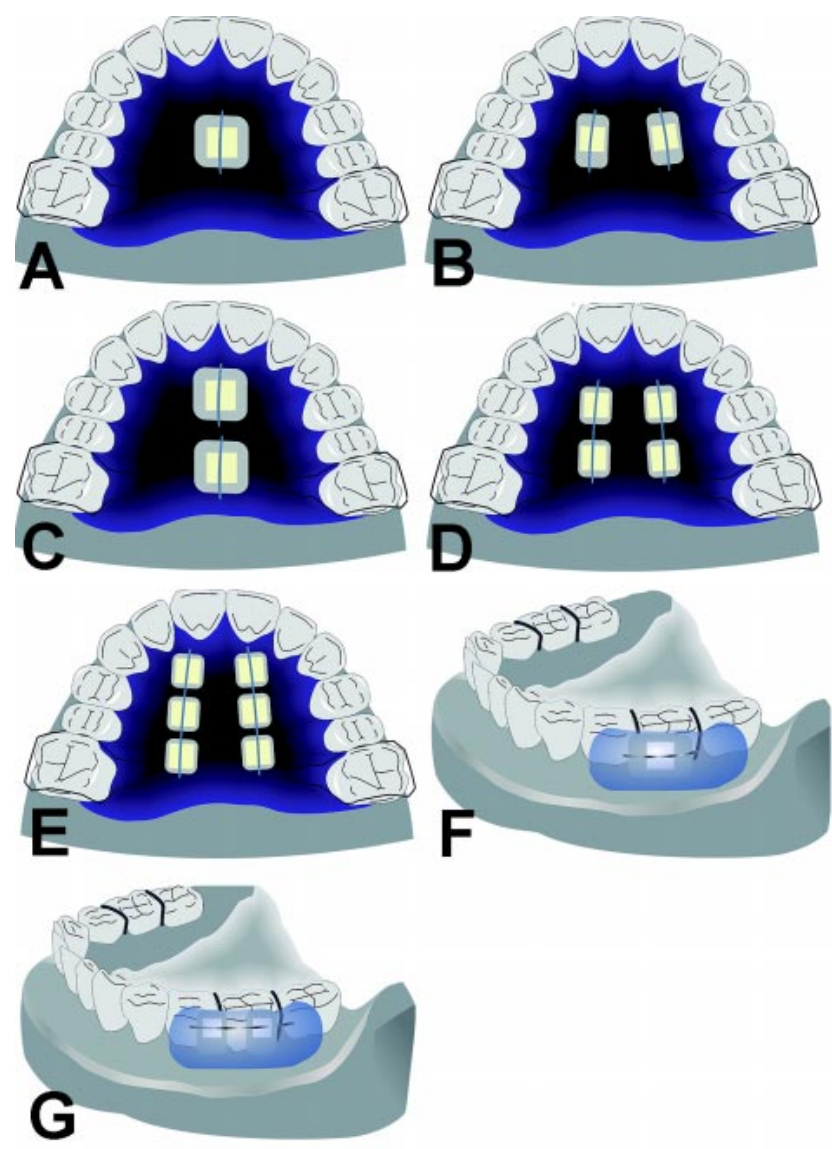

Fig. 2. Distribución de especímenes en los dispositivos removibles a. Dispositivo palatino con 1 espécimen, b. Dispositivo palatino con 2 especímenes (derecha e izquierda), c. Dispositivo palatino con 2 especímenes (anterior y posterior), d. Dispositivo palatino con 4 especímenes, e. Dispositivo palatino con 6 especímenes, f. Dispositivo mandibular con 1 espécimen, g. Dispositivo mandibular con 2 especímenes.

La ubicación del dispositivo influye en la pérdida del esmalte ocasionada por los desafíos erosivos, siendo preferido el uso de dispositivos palatinos tanto por investigadores y participantes. Mendonça et al. (2019) encontraron que la ubicación del dispositivo no influyó en la capacidad de remineralización de la saliva sobre el esmalte erosionado o en el efecto protector de la saliva contra la desmineralización erosiva inicial (Mendonça et al., 2017). Sin embargo, Jordão et al. encontraron que el dispositivo palatino fue más cómodo y produjo mayor pérdida de esmalte por lo que podrían imitar desafíos erosivos más severos, en comparación con el dispositivo mandibular.

También se ha utilizado el diseño de prótesis total en pacientes edéntulos para determinar la in- 
fluencia de la ubicación y forma de ingerir una bebida (Fig. 3). Se encontró la mayor pérdida de tejido en las superficies oclusales de molares inferiores, superficies palatinas en zonas anterosuperiores y superficies linguales en zonas anteroinferiores, reflejando la distribución que se observa clínicamente en pacientes con erosión. Por otro lado, la forma en que los pacientes ingieren las bebidas juega un rol importante en el desarrollo de la erosión ya que mantener la bebida en la boca en comparación de solamente pasarla, aumenta la tasa de desmineralización (Ruben et al.).
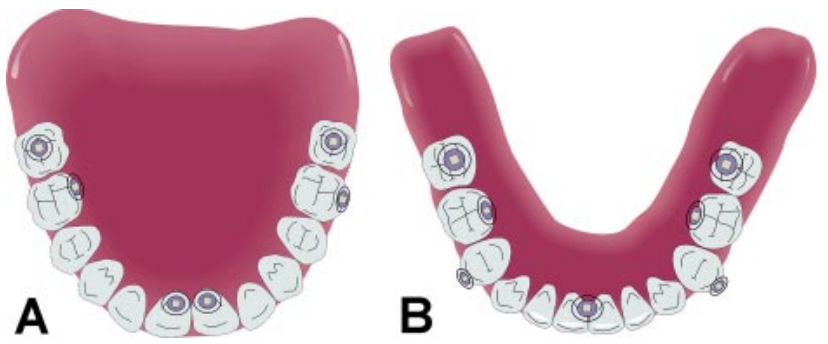

Fig. 3. Distribución de especímenes en prótesis total para ensayos in situ a. Superior, b. Inferior.

Agentes erosivos. En la literatura encontramos diferentes agentes erosivos, con mayor frecuencia se utiliza el ácido clorhídrico (HCL) para simular eventos asociados a menudo con trastornos alimentarios (Young \& Tenuta). Los datos mostraron que hasta $30 \mathrm{~s}$ de inmersión es el tiempo óptimo para el desarrollo de lesiones de erosión iniciales (Mendonça et al., 2017, 2019) y para lesiones erosivas avanzadas de 2 a 5 minutos (Rios et al., 2019; Santos et al.; Jordão et al.). El ácido cítrico ( $\mathrm{pH}$ 3.6) se utiliza por ser el tipo de ácido más común en las bebidas ácidas (Shellis et al.). El tiempo de inmersión en ácido cítrico para el desarrollo de lesiones erosivas iniciales es hasta 2 min y para lesiones erosivas avanzadas de 2 a 5 minutos (Almeida). Además se utilizan como sustancias control Coca cola $₫$ y agua destilada con un tiempo de inmersión promedio de 1 minuto y medio a 5 minutos (West et al., 2011; Shellis et al.; Young \& Tenuta; Wiegand \& Attin, 2011; Almeida; Kensche et al.; Ablal et al.).

Se han utilizado dos maneras de exponer al agente erosivo, administrando directamente la sustancia erosiva a la boca del participante o mediante una exposición extraoral al agente erosivo (West et al., 2011; Shellis et al.; Huysmans et al.; Almeida; Scatena et al.; Jordão et al.).
Ciclos erosivos. No se ha determinado un ciclo erosivo estándar, sin embargo encontramos que uno de los ciclos más frecuentes es: tiempo de inmersión de $30 \mathrm{~s}$ a 5 minutos, 4 inmersiones diarias para simular la ingesta regular de individuos considerados de alto riesgo de erosión dental (Rios et al., 2006), de 1 a 5 días. Si el participante recibe diferentes sustancias de experimentación se requiere un periodo de ventana (wash out) de 3 a 10 días con el objetivo de evitar efectos cruzados (Hara et al., 2006; Acuña et al.; Shellis et al.; da Silva et al.).

Ensayos / test empleado. Los métodos más utilizados en estudios in vitro e in situ para evaluar potencial erosivo son: análisis químicos de liberación de minerales y dureza superficial del esmalte para la erosión temprana, y para estadios de erosión avanzada se utilizan perfilometría y microradiografía de la superficie. Los cambios morfológicos en el tejido dental erosionado generalmente se han caracterizado por la microscopía electrónica de barrido (Gregg et al.). Otros metodos utilizados son fluorescencia cuantitativa inducida por luz (QLF) y tomografia de coherencia optica (OCT) (Schlueter et al., 2011; Joshi et al., 2016; Katkar et al., 2018).

Análisis químico de minerales disueltos. Es utilizado para cuantificar la liberación de iones de calcio y fosfato mediante una solución ácida (Schlueter et al.). Calcio y fosfato se pueden analizar colorimétricamente o mediante la espectrofotometría de absorción atómica para el análisis de calcio evitando la interferencia del fosfato (Joshi et al.). Se complementa con el análisis químico $(\mathrm{pH}$, acidez titulable, capacidad buffer) de las sustancias evaluadas.

Microdureza superficial (MS). La pérdida de dureza se mide por la resistencia de un sustrato a la deformación plástica luego de la penetración de un indentador. La MS se mide con un penetrador de diamante Knoop o Vickers, que son romboidales y tetrapiramidales, respectivamente (Schlueter et al.).Se puede analizar la diferencia de microdureza superficial (DMS= MSfinal - MSinicial) o el porcentaje de pérdida de microdureza (\% PPD $=[($ MSfinal MSinicial) / MSinicial] * 100) (Rios et al., 2009; Acuña et al.).

Perfilometría de superficie. Cuantifica la pérdida de tejido dental en relación con un área de referencia no tratada. El perfilómetro se puede utilizar para medir el contorno de la superficie, el perfil y la rugosidad. 
La superficie de una muestra se escanea para generar un perfil bidimensional o tridimensional, utilizando un dispositivo de medición de contacto (aguja de acero o diamante) o no contacto (basados en luz). Se prefiere la de no contacto porque el lector del equipo no daña la superficie de la muestra pudiendo alterar los resultados (Schlueter et al.; Joshi et al.).

La microrradiografía. Cuantifica la pérdida de minerales basada en la atenuación de la radiación de rayos $\mathrm{X}$ que transmite el tejido duro dental (West et al., 2011; Shellis et al.; Schlueter et al.).

Microscopía electrónica de barrido. Es una caracterización cualitativa de superficie que permite visualizar las alteraciones superficiales después de un desafío erosivo. En el esmalte se observa una apariencia de panal similar a un patrón de grabado con ácido fosfórico con exposición de prismas y en dentina, apertura de túbulos dentinarios dependiendo de la gravedad del desafío erosivo (West et al., 2011; Shellis et al. ; Schlueter et al.; Joshi et al.).

\section{Fluorescencia cuantitativa inducida por luz (QLF):} Es un método óptico que se basa en la autofluorescencia del esmalte, que disminuye con la pérdida del contenido mineral. Las áreas desmineralizadas aparecen más oscuras (Joshi et al.).

Tomografia de coherencia optica (OCT): Es una técnica no invasiva que proporciona imágenes transversales de estructuras biológicas basadas en las diferencias en las propiedades ópticas de los tejidos, utiliza ondas de luz infrarroja que se reflejan en la microestructura interna. Las áreas erosionadas reflejan una mayor intensidad de luz (Schlueter et al.; Katkar et al.).

CONCLUSIONES. Los modelos in situ permiten estudiar diferentes metodologías de erosión dental en estadíos tempranos y avanzados, además de conocer el proceso de remineralización con ciclos erosivos exclusivos para erosión. Asimismo, permite tener resultados rápidos y de menor costo en comparación con estudios clínicos sin afectar la dentición natural.

ACUÑA, F. S. M.; TAY-CHU-JON, L. Y. \& DELGADOCOTRINA, L. In situ models to evaluate erosive potential of beverages. Int. J. Odontostomat., 15(3):788-794, 2021.

ABSTRACT: There are several methodologies in vitro, in situ and in vivo to evaluate the erosive potential of different drinks such as natural fruit juices, processed juices, alcoholic drinks, functional drinks or products and techniques for the control of this pathology. The in situ models allow tissues evaluated to develop in a natural environment, in the presence of saliva and acquired pellicle; in addition, the specimens can be removed to analyze their mechanical, physical and biological properties in a quantitative and qualitative way. The objective of this literature review was to present the different in situ models to evaluate the erosive potential of beverages on dental tissues. The criteria for the selection of participants, substrate, intraoral devices, erosive agents, erosive cycles and tests or tests used will be developed.

KEY WORDS: tooth erosion; beverages; in situ.

\section{REFERENCIAS BIBLIOGRÁFICAS}

Ablal, M. A.; Milosevic, A.; Preston, A. J. \& Higham, S. M. A novel approach to study in situ enamel erosion and abrasion lesions. $J$ Dent., 59:78-85, 2017.

Acuña, S. M.; Tay Chu Jon, L. Y.; Ccahuana, R. A. \& Delgado, L. Potencial erosivo de jugos de frutas amazónicas. Rev. Estomatol. Herediana, 30(3):187-95, 2020. Disponible en: https:// revistas.upch.edu.pe/index.php/REH/article/view/3821/4301

Almeida, M. C. Estabelecimento de Protocolos in vitro e In situ para Estudos de Erosão Dentária. Tesis. Bauru, Faculdade de Odontologia de Bauru, 2016

Amaechi, B. T. \& Higham, S. M. Eroded enamel lesion remineralization by saliva as a possible factor in the site-specificity of human dental erosion. Arch. Oral Biol., 46(8):697-703, 2001.

Amaechi, B. T.; Higham, S. M. \& Edgar, W. M. Factors influencing the development of dental erosion in vitro: enamel type, temperature and exposure time. J. Oral Rehabil., 26(8):62430,1999.

Amaechi, B. T.; Higham, S. M. \& Edgar, W. M. Influence of abrasion in clinical manifestation of human dental erosion. J. Oral Rehabil., 30(4):407-13, 2003.

Attin, T. \& Wegehaupt, F. J. Methods for assessment of dental erosion. Monogr. Oral Sci., 25:123-42, 2014.

Buzalaf, M.; Magalhães, A. C. \& Rios, D. Prevention of erosive tooth wear: targeting nutritional and patient-related risks factors. $\mathrm{Br}$. Dent. J., 224(5):371-8, 2018.

Chan, A. S.; Tran, T.; Hsu, Y. H.; Liu, S. \& Kroon, J. A systematic review of dietary acids and habits on dental erosion in adolescents. Int. J. Paediatr. Dent., 30(6):713-33, 2020.

da Silva, C. V.; Ramos-Oliveira, T. M.; Mantilla, T. F. \& de Freitas, P. $\mathrm{M}$. Frequency of application of $\mathrm{AmF} / \mathrm{NaF} / \mathrm{SnCl} 2$ solution and its potential in controlling human enamel erosion progression: an in situ study. Caries Res., 51(2):141-8, 2017.

de Souza, B. M.; Santi, L.; de Souza Silva, M.; Buzalaf, M. \& Magalhães, A. C. Effect of an experimental mouth rinse containing $\mathrm{NaF}$ and $\mathrm{TiF}(4)$ on tooth erosion and abrasion in situ. J. Dent., 73:45-9, 2018.

Field, J. C.; Waterhouse, P. J. \& German, M. J. The early erosive and abrasive challenge: a profilometric, electron microscopic and microhardness study using human, bovine and ovine enamel. Eur. J. Prosthodont. Restor. Dent., 25(2):93-100, 2017.

Gregg, T.; Mace, S.; West, N. X. \& Addy, M. A study in vitro of the abrasive effect of the tongue on enamel and dentine softened by acid erosion. Caries Res., 38(6):557-60, 2004.

Hara, A. T.; Ando, M.; González-Cabezas, C.; Cury, J. A.; Serra, M. C. \& Zero, D. T. Protective effect of the dental pellicle against erosive challenges in situ. J. Dent. Res., 85(7):612-6, 2006. 
Hara, A. T.; Barlow, A. P.; Eckert, G. J. \& Zero, D. T. Novel in-situ longitudinal model for the study of dentifrices on dental erosionabrasion. Eur. J. Oral Sci., 122(2):161-7, 2014.

Hooper, S. M.; Newcombe, R. G.; Faller, R.; Eversole, S.; Addy, M. \& West, N. X. The protective effects of toothpaste against erosion by orange juice: studies in situ and in vitro. J. Dent., 35(6):476-81, 2007.

Hooper, S.; Seong, J.; Macdonald, E.; Claydon, N.; Hellin, N.; Barker, M. L.; He, T. \& West, N. X. A randomized in situ trial, measuring the anti-erosive properties of a stannous-containing sodium fluoride dentifrice compared with a sodium fluoride/potassium nitrate dentifrice. Int. Dent. J., 64 Suppl. 1:35-42, 2014.

Huysmans, M. C.; Chew, H. P. \& Ellwood, R. P. Clinical studies of dental erosion and erosive wear. Caries Res., 45 Suppl. 1:60-8, 2011.

João-Souza, S. H.; Bezerra, S.; de Freitas, P. M.; de Lima, N. B.; Aranha, A.; Hara, A. T. \& Scaramucci, T. In situ evaluation of fluoride-, stannous- and polyphosphate-containing solutions against enamel erosion. J. Dent., 63:30-5, 2017.

Jordão, M. C.; lonta, F. Q.; Bergantin, B.; Mendonça, F. L.; Santos, N.; Honório, H. M.; Oliveira, T. M. \& Rios, D. Influence of mandibular and palatal intraoral appliances on erosion in situ study outcome. J. Appl. Oral Sci., 27:e20180153, 2019.

Joshi, M.; Joshi, N.; Kathariya, R.; Angadi, P. \& Raikar, S. Techniques to evaluate dental erosion: a systematic review of literature. J. Clin. Diagn. Res., 10(10):ZE01-ZE07, 2016.

Katkar, R. A.; Tadinada, S. A.; Amaechi, B. T. \& Fried, D. Optical coherence tomography. Dent. Clin. North Am., 62(3):421-34, 2018.

Kensche, A.; Buschbeck, E.; König, B.; Koch, M.; Kirsch, J.; Hannig, C. \& Hannig, M. Effect of fluoride mouthrinses and stannous ions on the erosion protective properties of the in situ pellicle. Sci. Rep., 9(1):5336, 2019.

Magalhães, A. C.; Rios, D.; Honório, H. M.; Delbem, A. C. \& Buzalaf, M. A. Effect of $4 \%$ titanium tetrafluoride solution on the erosion of permanent and deciduous human enamel: an in situlex vivo study. J. Appl. Oral Sci., 17(1):56-60, 2009.

Mendonça, F. L.; Jordão, M. C.; Ionta, F. Q.; Buzalaf, M.; Honório, H. M.; Wang, L. \& Rios, D. In situ effect of enamel salivary exposure time and type of intraoral appliance before an erosive challenge. Clin. Oral Investig., 21(8):2465-71, 2017.

Mendonça, F. L.; Jordão, M. C.; Val, P. P.; de Alencar, C. R.; Bassoto, M. A.; Honório, H. M.; Magalhães, A. C.; Buzalaf, M. A.; da Silva, T. C. \& Rios, D. Eroded enamel rehardening using two intraoral appliances designs in different times of salivary exposure. J. Clin. Exp. Dent., 11(12):e1127-32, 2019.

Moda, M. D.; Fagundes, T. C.; Bresciani, E.; Briso, A. \& Dos Santos, P. $\mathrm{H}$. Comparison of in vitro erosion protocols in bovine teeth to simulate natural erosion lesion: analysis of mechanical properties and surface gloss. J. Appl. Oral Sci., 27:e20180107, 2019.

Mullan, F.; Austin, R. S.; Parkinson, C. R. \& Bartlett, D. W. An in-situ pilot study to investigate the native clinical resistance of enamel to erosion. J. Dent., 70:124-8, 2018.

Rios, D.; Honório, H. M.; Magalhães, A. C.; Delbem, A. C.; Machado, M. A.; Silva, S. M. \& Buzalaf, M. A. Effect of salivary stimulation on erosion of human and bovine enamel subjected or not to subsequent abrasion: an in situlex vivo study. Caries Res., 40(3):218-23, 2006.

Rios, D.; Honório, H. M.; Magalhães, A. C.; Silva, S. M.; Delbem, A. C.; Machado, M. A. \& Buzalaf, M. A. Scanning electron microscopic study of the in situ effect of salivary stimulation on erosion and abrasion in human and bovine enamel. Braz. Oral Res., 22(2):132-8, 2008.

Rios, D.; Honório, H. M.; Magalhães, A. C.; Wiegand, A.; de Andrade Moreira Machado, M. A. \& Buzalaf, M. A.. Light cola drink is less erosive than the regular one: an in situlex vivo study. J. Dent., 37(2):163-6, 2009

Rios, D.; Oliveira, G. C.; Zampieri, C. R.; Jordão, M. C.; Dionisio, E. J.; Buzalaf, M.; Wang, L. \& Honório, H. M. Resin-Based Materials Protect Against Erosion/Abrasion-a Prolonged In situ Study. Oper. Dent., 44(3):302-11, 2019.
Ruben, J.; Truin, G. J.; Bronkhorst, E. M. \& Huysmans, M. C. A new in situ model to study erosive enamel wear, a clinical pilot study. J. Dent., 57:32-7, 2017.

Santos, N. M.; Jordão, M. C.; lonta, F. Q.; Mendonça, F. L.; Di Leone, C.; Buzalaf, M.; Oliveira, T. M.; Honório, H. M.; Cruvinel, T. \& Rios, D. Impact of a simplified in situ protocol on enamel loss after erosive challenge. PLoS One, 13(5):e0196557, 2018.

Scatena, C.; de Mesquita-Guimarães, K.; Galafassi, D.; Palma-Dibb, R. G.; Borsatto, M. C. \& Serra, M. C. Effects of a potentially erosive antiasthmatic medicine on the enamel and dentin of primary teeth: An in situ study. Microsc. Res. Tech., 81(9):1077-83, 2018.

Schlueter, N. \& Luka, B. Erosive tooth wear - a review on global prevalence and on its prevalence in risk groups. Br. Dent. J., 224(5):364-70, 2018

Schlueter, N.; Hara, A.; Shellis, R. P. \& Ganss, C. Methods for the measurement and characterization of erosion in enamel and dentine. Caries Res., 45 Suppl. 1:13-23, 2011.

Shellis, R. P.; Ganss, C.; Ren, Y.; Zero, D. T. \& Lussi, A. Methodology and models in erosion research: discussion and conclusions. Caries Res., 45 Suppl. 1:69-77, 2011.

Turssi, C. P.; Hara, A. T.; Amaral, F. L.; França, F. M. \& Basting, R. T. Calcium lactate pre-rinse increased fluoride protection against enamel erosion in a randomized controlled in situ trial. J. Dent., 42(5):534-9, 2014

Turssi, C. P.; Messias, D. F.; Corona, S. M. \& Serra, M. C. Viability of using enamel and dentin from bovine origin as a substitute for human counterparts in an intraoral erosion model. Braz. Dent. J., 21(4):3326, 2010.

Vieira Pedrosa, B. R. \& de Menezes, V. A. Prevalence of erosive tooth wear and related risk factors in adolescents: an integrative review. J. Dent. Child. (Chic.), 87(1):18-25, 2020.

Warreth, A.; Abuhijleh, E.; Almaghribi, M. A.; Mahwal, G. \& Ashawish, A. Tooth surface loss: A review of literature. Saudi Dent. J., 32(2):5360, 2020.

West, N. X.; Davies, M. \& Amaechi, B. T. In vitro and in situ erosion models for evaluating tooth substance loss. Caries Res., 45 Suppl. 1:43-52, 2011

West, N. X.; He, T.; Hellin, N.; Claydon, N.; Seong, J.; Macdonald, E.; Farrell, S.; Eusebio, R. \& Wilberg, A. Randomized in situ clinical trial evaluating erosion protection efficacy of a $0.454 \%$ stannous fluoride dentifrice. Int. J. Dent. Hyg., 17(3):261-7, 2019.

West, N. X.; Hughes, J. A.; Parker, D.; Weaver, L. J.; Moohan, M.; De'Ath, J. \& Addy, M. Modification of soft drinks with xanthan gum to minimise erosion: a study in situ. Br. Dent. J., 196(8):478-81; discussion 467, 2004.

Wiegand, A. \& Attin, T. Design of erosion/abrasion studies-insights and rational concepts. Caries Res., 45 Suppl. 1:53-9, 2011.

Wiegand, A. \& Attin, T. Randomised in situ trial on the effect of milk and CPP-ACP on dental erosion. J. Dent., 42(9):1210-5, 2014.

Yassen, G. H.; Platt, J. A. \& Hara, A. T. Bovine teeth as substitute for human teeth in dental research: a review of literature. J. Oral Sci., 53(3):273-82, 2011.

Young, A. \& Tenuta, L. M. Initial erosion models. Caries Res., 45 Suppl. 1:33-42, 2011.

Dirección para correspondencia:

Shareen Acuña

Posgrado de Estomatología

Av. Salaverry 2475 San Isidro

Lima - PERÚ

E-mail: shareen.acuna@upch.pe 